\title{
EXPLORANDO A DISTRIBUIÇÃO DO FINANCIAMENTO PARTIDÁRIO PELAS COLIGAÇÕES DE DEPUTADOS FEDERAIS E ESTADUAIS NAS ELEIÇÕES PARANAENSES DE 2018
}

Luiz Fernando Zelinski ${ }^{1}$

Eduardo Soncini Miranda ${ }^{2}$

\begin{abstract}
Resumo
Este trabalho investiga quais são os efeitos do Financiamento Partidário mensurado pela soma do Fundo Especial de Financiamento de Campanha (FEFC), do tradicional Fundo Partidário e das doações privadas que têm o partido como agente intermediador da doação. A questão central do artigo é como as coligações de partidos realizam a distribuição do Financiamento Partidário entre seus candidatos para o alcance de cadeiras e qual o impacto dessa modalidade de financiamento em contribuir para o desempenho eleitoral de candidatos? Para tal, exploramos 416 candidaturas para deputados federais e 706 para deputados estaduais nas eleições paranaenses de 2018, estando elas agregadas em 12 e 14 coligações respectivamente. A fonte de dados é o Repositório de Dados Eleitorais do Tribunal Superior Eleitoral (TSE). $\mathrm{O}$ artigo inova ao testar a aplicabilidade do Coeficiente de Gini para mensurar em maior ou menor grau a distribuição/concentração do Financiamento Partidário nas mãos de candidatos para o alcance do sucesso eleitoral. A esse Coeficiente, somam-se demais métodos e técnicas já consolidados na literatura do financiamento eleitoral, como as análises de regressão linear simples e multivariada. Os resultados da exploração apontam que a distribuição do Financiamento Partidário ocorre de maneira diferente entre os cargos; determinantes do financiamento ligados ao perfil dos candidatos também operam de maneiras diferentes; o Financiamento Partidário impacta mais no alcance de votos pelos candidatos ao cargo de deputado federal, do que ao cargo de deputado estadual.
\end{abstract}

Palavras-chave: financiamento eleitoral; eleições 2018; partidos; coligações; Coeficiente de Gini.

\section{INTRODUÇÃO}

A literatura da Ciência Política que acerca o debate sobre o financiamento eleitoral de campanhas tem sido unânime em afirmar que dinheiro influencia, determinando ou contribuindo, para o desempenho e o sucesso eleitoral de candidatos (BORBA; CERVI, 2017; CERVI, 2010; HOROCHOVSKI; JUNCKES; ZELINSKI, 2018; MANCUSO, 2015; MANCUSO; SPECK, 2015a; SAMUELS, 2001; SPECK; CERVI, 2016). No mesmo sentido, tem-se investigado, quais os determinantes do financiamento (MANCUSO; SPECK, 2015b; SACCHET; SPECK, 2012; SPECK;

\footnotetext{
${ }^{1}$ Doutorando (PPGCP-UFPR), fernandozelinski@ufpr.br, ORCID: https://orcid.org/0000-0002-5757-8239.

${ }^{2}$ Doutor em Ciência Política (PPGCP-UFPR), eduardomiranda@ufpr.br, ORCID: https://orcid.org/0000-0002-4573-7116
} 
MANCUSO, 2014; ZELINSKI; EDUARDO, 2019). Em geral, esses estudos orientam-se por aprofundar, de que maneira elementos que caracterizam o perfil individual do candidato, tal qual gênero, cor da pele, grau de instrução, capital político e/ou simbólico, entre outros, criam condições para o acesso ao financiamento.

Os estudos, em sua maioria, centralizam as análises no indivíduo (candidato) e, em menor grau, nos partidos políticos. Com exceção aos trabalhos que utilizam a análise de redes sociais (ARS), para a exploração da estrutura de financiamento constituída pelos relacionamentos entre doadores (pessoas físicas, jurídicas e partidos) e receptores (candidatos, comitês e partidos) (GEARA et al., 2018; HOROCHOVSKI et al., 2015, 2016; JUNCKES et al., 2019; SILVA et al., 2017). No segundo caso, a busca é compreender como os partidos captam e distribuem seus recursos.

A questão central deste artigo é como as coligações de partidos realizam a distribuição do Financiamento Partidário entre seus candidatos para o alcance de cadeiras e qual o impacto dessa modalidade de financiamento em contribuir para o desempenho eleitoral de candidatos? Outros questionamentos contribuem para o aprimoramento das explorações, tais como: pressupondo que os partidos distribuem seus recursos a fim de otimizar suas chances de sucesso eleitoral, quais os perfis de candidatos possuem maior acesso ao Financiamento Partidário? Em outras palavras, elementos de cunho individual do candidato (determinantes do financiamento) operam como variáveis que promovem acesso a esse recurso? É possível identificar as estratégias das coligações que possibilitem o sucesso eleitoral, a partir de maior ou menor concentração/distribuição do elemento que está sendo estudado? A concentração desse financiamento em poucos candidatos ou a distribuição em muitos candidatos contribui para maiores chances de sucesso eleitoral?

Inspirado pelo trabalho de Bolognesi et al. (2018), este artigo, de maneira exploratória, testa a aplicabilidade do Coeficiente de Gini sobre o Financiamento Partidário e aliado às demais variáveis, métodos e técnicas, contribui para a compreensão da lógica assertiva das coligações em distribuir recursos financeiros para seus candidatos. Não obstante, contribui com a literatura sobre o Financiamento Eleitoral de Campanhas, ao dar um pontapé inicial dentro de nossa agenda nos estudos, que centralizam os partidos e coligações de partidos, como ente distribuidor dos recursos financeiros; com a oferta de elementos empíricos por meio de dados fidedignos; e com o aprimoramento metodológico, ao promover uma melhor compreensão do fenômeno.

As eleições de 2018 tem a característica de ser a primeira em que o aporte de financiamento público é maior que o de financiamento privado. O recurso público supera o total de 2 bilhões de 
reais, cerca de $66,6 \%$ do total movimentado, conforme dados do $\operatorname{TSE}^{3}$. Nesse sentido, vale dizer que entre as eleições de 1994 e 2014, a principal fonte de financiamento foi a privada, advinda, sobretudo, de doações empresariais (MANCUSO; HOROCHOVSKI; CAMARGO, 2018; ZELINSKI; EDUARDO, 2019).

Essa inversão na lógica da estrutura/matriz do financiamento cria um fenômeno novo e que centraliza maior poder de tomada de decisão, quanto ao direcionamento dos recursos financeiros, nas mãos dos partidos políticos e das coligações. Entretanto, em um momento no qual as eleições de 2018 foram as últimas com a permissão da formação de coligações para as disputas proporcionais ${ }^{4}$, o que esperar desses atores a partir das eleições de 2020, quando a formação de coligações não será mais possível?

Para esta exploração, a fonte de dados é o Repositório de Dados Eleitorais do Tribunal Superior Eleitoral. O recorte escolhido é o das eleições proporcionais paranaenses de 2018, em que investigamos 416 candidaturas para o cargo de deputado federal e 706 candidaturas para o cargo de deputado estadual, estando elas agregadas em 12 e 14 coligações respectivamente.

O trabalho está dividido como segue: na próxima seção apresentamos o método de investigação, fonte de dados e o recorte escolhido, bem como do processo de construção do banco de dados e das variáveis que serão utilizadas nas análises. A seguir, exploramos e apresentamos os dados relativos às coligações, candidatos, montantes financeiros e cadeiras conquistadas, além da análise estatística sobre os determinantes do Financiamento Partidário e do impacto deste financiamento na conquista de votos. Discutimos de maneira breve os resultados e tecemos algumas considerações, a fim de consolidar uma agenda de estudo mais assertiva.

\section{MÉTODO, FONTE E RECORTE}

Este trabalho tem como fonte de dados o Repositório de Dados Eleitorais do Tribunal Superior Eleitoral (TSE) ${ }^{5}$. Por meio do repositório, coletamos, mineramos, categorizamos e preparamos um

3 TSE, Estatísticas Eleitorais. Aba de prestação de contas. Disponível em: < http://www.tse.jus.br/eleicoes/estatisticas/estatisticas-eleitorais >. Acesso em: 27/06/2020

4 Senado aprova cláusula de barreira a partir de 2018 e fim da coligação para 2020. Disponível em: < https://www12.senado.leg.br/noticias/materias/2017/10/03/aprovado-fim-das-coligacoes-em-eleicoes-proporcionais-apartir-de-2020 >. Acesso em: 27/06/2020

5 Repositório de Dados Eleitorais do Tribunal Superior Eleitoral (TSE) < http://www.tse.jus.br/eleicoes/estatisticas/repositorio-de-dados-eleitorais-1/repositorio-de-dados-eleitorais >. 
banco de dados que é composto de informações sobre o perfil dos candidatos (gênero, grau de instrução, cor da pele, etc...), soma dos valores de doações eleitorais (fonte e origem do financiamento) e soma de votos conquistados pelos candidatos.

A partir disso, agregamos ao nosso banco de dados variáveis na forma de indicadores, que se referem à cálculos sobre o financiamento e o voto de cada candidato. Tais variáveis permitem a operacionalização de modelos estatísticos de regressão linear simples e multivariada. Esses indicadores, não só viabilizam a construção de modelos estatísticos para uma análise quantitativa, bem como comparativa entre distritos eleitorais de proporções/magnitudes bastante desiguais, o que enseja uma agenda de trabalhos que tenha as demais unidades federativas do Brasil como recorte. $\mathrm{O}$ banco de dados ${ }^{6}$ com o recorte estudado neste trabalho está disponível em repositório online do autor para replicação dos estudos.

Esses indicadores são chamados de Indicadores Percentuais (IP) e seu sufixo indica qual variável está sendo calculada. O IP_FINANCIAMENTO ${ }^{7}$ é basicamente o cálculo do percentual de financiamento - ou da modalidade de financiamento - do candidato em relação a soma total do financiamento dos demais candidatos ao mesmo cargo. Utilizamos também o IP_VOTO, que é o cálculo do percentual de votos recebido pelo candidato, partido ou coligação em relação a soma dos demais que competiram ao mesmo cargo.

Existem algumas modalidades que se dividem entre financiamento público e privado. $\mathrm{O}$ financiamento público é de controle dos partidos políticos e composto por duas fontes, sendo uma o Fundo Especial de Financiamento de Campanhas (FEFC), que segundo dados do $\mathrm{TSE}^{8}$, nas eleições de 2018 foi aportado um total superior a 1,7 bilhão de reais. A outra modalidade é o tradicional fundo partidário, este em menor volume, porém também significativo, alcançando cerca de 300 milhões de reais.

Nas modalidades de financiamento privado, extinguido o financiamento por empresas desde as eleições de $2016^{9}$, temos entre as principais fontes o financiamento por pessoa física, a autodoação (quando candidatos investem seu próprio patrimônio em sua campanha) e de demais candidatos, entre outros menos expressivos. À pessoa física é permitido a doação direta para candidatos e partidos

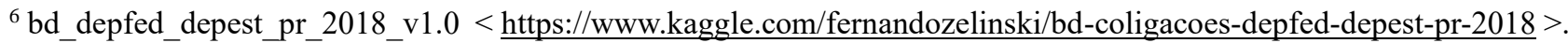

7 O IP_FINACIAMENTO será sempre alguma modalidade de financiamento, podendo ser público, privado, partidário, entre outros.

${ }^{8}$ Partidos políticos receberam R\$ 1,7 bilhão do Fundo Eleitoral em 2018: < http://www.tse.jus.br/imprensa/noticiastse/2019/Janeiro/partidos-politicos-receberam-r-1-7-bilhao-do-fundo-eleitoral-em-2018 >. Acesso em: 12/05/2020

${ }^{9}$ A proibição de doações por pessoas jurídicas é estabelecida a partir da Lei $\mathrm{n}^{\circ} 13.165 / 15$, que é a consolidação do julgamento da Ação Direta de Inconstitucionalidade (ADI) 4.650. Para mais, vide Miranda \& Zelinski (2019); Zelinski, \& Eduardo (2019); Zelinski, Miranda e Eduardo (2019).
} 
políticos desde que respeitado o limite de $10 \%$ da sua renda bruta auferida e declarada à Receita Federal no ano anterior ao da eleição. Quando a pessoa doa para o partido político, o partido pode ou não repassar para um candidato. Sendo assim, em caso de repasse do partido para um candidato, o que temos é uma doação originária de pessoa física que foi intermediada pelo partido político.

Para este material, utilizaremos basicamente quatro tipos/modalidades de financiamento, são eles:

I. Financiamento total, que se refere a soma de todo e qualquer tipo de doação recebida pelo candidato;

II. Financiamento público, que se refere a soma do FEFC + Fundo Partidário;

III. Financiamento privado, que se refere a soma de toda e qualquer doação em que a fonte seja uma pessoa física (vale para candidatos, como no caso das autodoações);

IV. Financiamento Partidário, que já explicado anteriormente, se refere a soma do FEFC + Fundo Partidário + doações privadas ${ }^{10}$ intermediadas pelos partidos políticos.

Outro indicador calculado e agregado ao banco de dados se refere ao Coeficiente de Gini. Calculamos o Coeficiente de Gini para mensurar o maior ou menor grau de concentração do Financiamento Partidário mediante a prestação de contas dos candidatos. Espera-se a partir da operacionalização dessa variável contribuir para melhor entender como partidos e coligações agem na dinâmica da distribuição e concentração de doações eleitorais.

Ainda que o banco de dados construído seja em nível nacional (alcançando todas as candidaturas de 2018 nas 27 unidades federativas), para um estudo de cunho exploratório, melhor manuseio dos dados e compreensão dos resultados, (além da proximidade dos autores com o fenômeno das eleições paranaenses) o nosso recorte é o das candidaturas ao cargo de deputado federal e deputado estadual nas eleições paranaenses de 2018. Foram analisados um total de 416 candidaturas para deputado federal que se distribuem em 12 coligações. As candidaturas para deputado estadual somam 706 e se dividem em 14 coligações. Os demais dados que se referem aos valores e a forma de distribuição passam a ser apresentados e descritos nas seções seguintes.

${ }^{10}$ O Financiamento Partidário é pouco afetado pelas doações privadas intermediadas pelos partidos. Em determinados casos o valor beira ao residual não ultrapassando $2 \%$ do valor total. 


\section{EXPLORAÇÃO E DISCUSSÃO SOBRE OS DADOS}

A Tabela 1Erro! Fonte de referência não encontrada. resume de maneira sistematizada por cargo informações relativas às coligações, os partidos que às compõem, total de candidatos eleitos e não eleitos, o montante em valores absolutos e médio do Financiamento Partidário declarados pelos candidatos e o Coeficiente de Gini calculado esse financiamento. 
Universidade Federal do Pa-.....

Curitiba, 20 a 21 de agosto de 2020

TABELA 1 - COLIGAÇÕES, CANDIDATOS, FINANCIAMENTO PARTIDÁRIO TOTAL, MÉDIO E COEFICIENTE DE GINI PARA OS CARGOS DE DEPTUADO FEDERAL E ESTADUAL

\begin{tabular}{|c|c|c|c|c|c|c|c|}
\hline \multicolumn{8}{|c|}{ DEPUTADO FEDERAL } \\
\hline COLIGAÇÃO & COMPOSIÇÃO DE PARTIDOS & ELEITO & NÃO ELEITO & TOT CAND & TOT FIN PARTIDÁRIO & MÉDIA FIN. PARTIDÁRIO & GINI \\
\hline COL_1 & MDB / PDT / SOLIDARIEDADE / PC do B & 3 & 40 & 43 & $\mathrm{R} \$ 6.043 .635,93$ & $\mathrm{R} \$ 140.549,67$ &, 836 \\
\hline COL_2 & NOVO & 0 & 16 & 16 & $\mathrm{R} \$ 178.486,56$ & $\mathrm{R} \$ 11.155,41$ &, 328 \\
\hline $\mathrm{COL}_{3} 3$ & $\mathrm{PCO}$ & 0 & 1 & 1 & $\mathrm{R} \$ 1.600,00$ & $\mathrm{R} \$ 1.600,00$ & - \\
\hline COL_4 & $\mathrm{PP} / \mathrm{PTB} / \mathrm{DEM}$ / PMN / PMB / PSB / PSDB / PROS & 8 & 36 & 44 & $\mathrm{R} \$ 21.028 .861,27$ & $\mathrm{R} \$ 477.928,67$ &, 664 \\
\hline COL_5 & PRB / PHS / AVANTE & 2 & 37 & 39 & $\mathrm{R} \$ 2.451 .818,27$ & $\mathrm{R} \$ 62.867,14$ &, 774 \\
\hline COL_6 & PRTB / PRP & 0 & 42 & 42 & $\mathrm{R} \$ 36.660,00$ & $\mathrm{R} \$ 872,86$ & 856 \\
\hline COL_ 7 & $\mathrm{PSD} / \mathrm{PSC}$ / PR / PPS / PODE & 10 & 35 & 45 & $\mathrm{R} \$ 17.843 .073,72$ & $\mathrm{R} \$ 396.512,75$ &, 724 \\
\hline COL_8 & PSL / PTC / PATRI & 3 & 38 & 41 & $\mathrm{R} \$ 290.200,00$ & $\mathrm{R} \$ 7.078,05$ & ,902 \\
\hline COL_9 & $\mathrm{PSOL} / \mathrm{PCB}$ & 0 & 29 & 29 & $\mathrm{R} \$ 78.755,91$ & $\mathrm{R} \$ 2.715,72$ & 000 \\
\hline COL_10 & PT & 3 & 30 & 33 & $\mathrm{R} \$ 4.120 .287,52$ & $\mathrm{R} \$ 124.857,20$ & 804 \\
\hline COL_11 & PV & 1 & 39 & 40 & $\mathrm{R} \$ 1.932 .069,16$ & $\mathrm{R} \$ 48.301,73$ & 829 \\
\hline COL_12 & REDE / DC / PPL & 0 & 43 & 43 & $\mathrm{R} \$ 117.814,19$ & $\mathrm{R} \$ 2.739,86$ & .578 \\
\hline TOTĀL & & 30 & 386 & 416 & R\$ 54.123.262,53 & $\mathbf{R} \$ 1.277 .179,05$ & \\
\hline \multicolumn{8}{|c|}{ DEPUTADO ESTADUAL } \\
\hline COLIGAÇÃO & COMPOSIČÃO DE PARTIDOS & ELEITO & NÃO ELEITO & TOT CAND & TOT FIN PARTIDÁRIO & MÉDIA FIN. PARTIDÁRIO & GINI \\
\hline COL_1 & MDB & 2 & 23 & 25 & R 1.614.263,15 & $\mathrm{R} \$$ 64.570,53 & ,664 \\
\hline COL_2 & PDT / SOLIDARIEDADE / PC do B & 2 & 27 & 29 & $\mathrm{R} \$ 748.035,00$ & $\mathrm{R} \$ 25.794,31$ &, 709 \\
\hline $\mathrm{COL}_{3} 3$ & PODE & 1 & 35 & 36 & $\mathrm{R} \$ 10.333,31$ & $\mathrm{R} \$ 287,04$ & .763 \\
\hline COL_4 & PP / PTB / DEM / PSDB / PSB & 14 & 65 & 79 & $\mathrm{R} \$ 7.085 .689,68$ & $\mathrm{R} \$ 89.692,27$ &, 793 \\
\hline COL_5 & PPS & 3 & 42 & 45 & $\mathrm{R} \$ 1.201 .277,06$ & $\mathrm{R} \$ 26.695,05$ &, 642 \\
\hline COL_6 & PRB / PHS / PR / AVANTE & 3 & 59 & 62 & $\mathrm{R} \$ 2.794 .038,91$ & $\mathrm{R} \$ 45.065,14$ &, 618 \\
\hline COL_7 & PROS / PMB / PMN & 3 & 70 & 73 & $\mathrm{R} \$ 1.958 .629,98$ & $\mathrm{R} \$ 26.830,55$ &, 875 \\
\hline $\mathrm{COL}_{-} 8$ & PRTB / PRP & 1 & 75 & 76 & $\mathrm{R} \$ 279.473,63$ & $\mathrm{R} \$ 3.677,28$ &, 787 \\
\hline $\mathrm{COL}_{-}^{-9}$ & $\mathrm{PSC} / \mathrm{PSD}$ & 10 & 58 & 68 & $\mathrm{R} \$ 4.775 .767,66$ & $\mathrm{R} \$ 70.231,88$ &, 666 \\
\hline COL_10 & PSL / PTC / PATRI & 8 & 57 & 65 & $\mathrm{R} \$ 548.828,32$ & $\mathrm{R} \$ 8.443,51$ & ,956 \\
\hline COL_11 & $\mathrm{PSOL} / \mathrm{PCB}$ & 0 & 21 & 21 & $\mathrm{R} \$ 28.819,40$ & $\mathrm{R} \$ 1.372,35$ & 057 \\
\hline $\mathrm{COL}_{-} 12$ & PT & 4 & 27 & 31 & $\mathrm{R} \$ 790.821,68$ & $\mathrm{R} \$ 25.510,38$ &, 719 \\
\hline COL_13 & PV & 2 & 55 & 57 & $\mathrm{R} \$ 170.722,02$ & $\mathrm{R} \$ 2.995,12$ &, 919 \\
\hline $\mathrm{COL}_{-} 14$ & REDE / DC / PPL & 1 & 38 & 39 & $\mathrm{R} \$ 92.088,71$ & $\mathrm{R} \$ 2.361,25$ &, 584 \\
\hline TOTAL & & 54 & 652 & 706 & $\mathrm{R} \$ 22.098 .788,51$ & $R \$ 393.526,66$ & \\
\hline
\end{tabular}

FONTE: o autor com dados do TSE (2020) 
Inicialmente, apontamos que a composição (ou não) de alianças entre os partidos não necessariamente se repetem entre os cargos e que, ao que parece, não levam em consideração o posicionamento do partido no espectro ideológico esquerda-direita. Observe o caso do MDB que, na disputa para deputado federal, coligou-se com PDT/SOLIDARIEDADE/PCdoB, e nas disputas para deputado estadual optou por competir de forma isolada. Caso parecido com o do PODE, que concorreu isoladamente para deputado estadual, mas coligou-se com PSD/PSC/PR/PPS para deputado federal. PT e PV nas duas disputas concorreram de forma isolada. Outras alianças, no entanto, se repetem entre os cargos, como o caso de REDE/DC/PPL e PSOL/PCB e PRTB/PRP. Já o NOVO e o PCO lançaram candidaturas apenas para deputado federal e de maneira isolada.

No que se refere ao Financiamento Partidário, os montantes envolvidos são bastante discrepantes entre as coligações. Em valores absolutos, a soma do montante entre todas as coligações é cerca de 2,5 vezes maior em candidaturas de deputado federal e na média cerca de 3,5. Para esse último, poderia ou não, o número de candidaturas refletir essa diferença. Não obstante, os valores médios entre candidatos eleitos e não eleitos (vide as Figuras Figura 5 Figura 6 no apêndice) refletem uma diferença muito maior. Sobre essa questão, trataremos nos parágrafos mais adiante. Ao olhar para os montantes envolvidos, o número de candidaturas e as diferenças entre eleitos e não eleitos, entendemos que se faz necessário tratar os dados de maneira mais apurada e que o valor médio por candidatura não é a melhor maneira de mensurar concentração/distribuição do Financiamento Partidário, assim sendo, mensuramos esse pela aplicação do Coeficiente de Gini.

Na Tabela 1 e nas Figura 1Figura 2 (no apêndice deste trabalho apresentam o Coeficiente de Gini e os valores absolutos e médios), no que se refere ao valor absoluto por coligação, maior ou menor aporte financeiro não reflete de maneira direta o modo como o recurso é distribuído. Para os casos das disputas ao cargo de deputado federal, é possível observar que as duas coligações que mais somam recursos absolutos e por média, COL_4 e COL_7, apresentam um padrão de distribuição razoavelmente similar, variando entre ,664 e ,724, o que para os padrões observados aqui é abaixo da média das demais outras coligações. Ao menos para as coligações que se apresentam como mais significativas. Ou seja, essas coligações, em que pese o montante aportado, bem como a quantia de candidatos, são aquelas que distribuem seus recursos de maneira mais igual entre seus candidatos.

Para o caso das disputas ao cargo de deputado estadual, os dados são menos inteligíveis e intuitivos, não sendo de fácil mensuração e percepção a lógica entre o Coeficiente de Gini e o montante financeiro aportado pelas coligações. No entanto, as coligações COL_4 e COL_9, são aquelas que aportam maior volume financeiro e com um grau razoável de diferença na distribuição 
de recursos, sendo o Gini igual a ,793 e ,666, respectivamente. A partir desses, mediante as coligações em que o volume financeiro aportado foi mais próximo, o Coeficiente de Gini se distribui de maneira a não refletir em algum grau uma lógica como: quem tem mais dinheiro distribui melhor, ou quem tem menos dinheiro concentra mais o recurso.

Nas Figura 3 Figura 4 (no apêndice), são apresentadas a relação entre Coeficiente de Gini a partir do financiamento partidário e o $\mathrm{N}$ de cadeiras conquistadas pelas coligações, em que é possível observar que independentemente do cargo, maior ou menor Gini, não significa sucesso eleitoral (sucesso aqui mensurado pelo $\mathrm{N}$ de cadeiras conquistadas). No entanto, vale observar que, para o cargo de deputado federal, duas coligações que conquistaram cadeiras e que apresentam menor grau de concentração de recursos são também aquelas que apresentam maior sucesso eleitoral, as coligações COL_4 e COL_7, oito e dez cadeiras, respectivamente. E com base na Tabela 1, são aquelas que realizaram maior aporte financeiro. Isso indica que, mediante uma quantia considerável de recursos financeiros, a distribuição desses recursos em um maior número de candidaturas assumese como a estratégia que possibilita maior sucesso eleitoral.

No que se refere à Figura 4, que trata sobre a relação entre Coeficiente de Gini a partir do Financiamento Partidário e o $\mathrm{N}$ de cadeiras conquistadas pelas coligações, que disputaram ao cargo de deputado estadual -, há uma relação bastante diferente da anterior. O sucesso eleitoral para as coligações com maior $\mathrm{N}$ de cadeiras não se agrupam em um polo ou outro da concentração de recursos. Vide o caso das coligações COL_4, COL_9 e COL_10, as quais conquistaram 14, 10 e 8 cadeiras e com variações no Coeficiente de Gini que vão de ,793, ,666 e ,955, respectivamente. Assim sendo, para as disputas ao cargo de deputado estadual, os dados sugerem que maior ou menor concentração dos recursos financeiros não refletem diretamente no $\mathrm{N}$ de cadeiras conquistadas.

As Figura 5 Figura 6 (no apêndice) apresentam os valores médios do Financiamento Partidário entre candidatos eleitos e não eleitos para cada um dos cargos investigados. No que se refere às disputas para o cargo de deputado federal, observa-se que a média do financiamento entre os candidatos eleitos (nas coligações que tiveram ao menos um candidato eleito) é muito próxima, vide o caso das coligações COL_1, COL_4, COL_7, COL_10 e COL_11. A única coligação que conseguiu eleger um candidato com uma média mais baixa que os demais é a COL_5. No caso da COL_8, que teve três candidatos eleitos, todos tiveram única e exclusivamente financiamento privado.

Quanto às disputas ao cargo de deputado estadual, a média dos candidatos eleitos apresenta uma variação um pouco maior. Ocorrem casos como o da COL_1, em que o financiamento médio dos eleitos se aproximou da casa dos 233 mil reais. No caso da COL_13, o financiamento médio foi 
um pouco maior que 5 mil reais. Entre as coligações COL_4, COL_5, COL_6 e COL_7, os valores são mais aproximados variando entre 140mil e 180 mil.

\subsection{EXPLORAÇÃO ESTATÍSTICA DOS DADOS}

Dentro do recorte explorado por este trabalho, alguns dados apontam que maior ou menor aporte financeiro, não reflete de maneira direta o modo como o recurso é distribuído. Ou seja, ter muito dinheiro não implica necessariamente em distribuí-lo de maneira mais equânime. Da mesma forma, uma quantia reduzida de aporte financeiro não significa destiná-lo a um número igualmente reduzido de candidatos. Ademais, vale ressaltar que, para o caso das disputas ao cargo de deputado federal, a distribuição do Financiamento Partidário em um maior número de candidaturas é a estratégia que possibilita maior sucesso eleitoral; para o caso das disputas ao cargo de deputado estadual, maior ou menor concentração dos recursos financeiros, não reflete diretamente no $\mathrm{N}$ de cadeiras conquistadas.

Porém, o que se viu é que há um maior aporte financeiro nas candidaturas eleitas. Logo, cabe perguntar: esses candidatos são eleitos por possuírem maior aporte financeiro? Para melhor compreensão do fenômeno, realizamos dois testes estatísticos que exploram as seguintes questões:

a) o Financiamento Partidário é distribuído de maneira estratégica pelas coligações para determinados perfis de candidatos a fim de criar/potencializar melhores condições de disputa?

b) a maior ou menor distribuição do Financiamento Partidário pela coligação, entre seus candidatos, resulta em maior ou menor quantia de votos pelos mesmos candidatos dentro da coligação?

O primeiro teste $\mathbf{T 1}$ mensura o grau de determinação que as variáveis (isoladas ou combinadas) relativas ao perfil do candidato contribuem no acesso ao Financiamento Partidário. O segundo teste T2 mensura o grau de determinação do Financiamento Partidário sobre o total de votos conquistado pelo candidato. Seu objetivo é demonstrar o quanto naquela coligação o Financiamento Partidário contribuiu em determinar o voto do candidato dentro da própria coligação. O Quadro 1 apresenta as variáveis e a sua descrição em nossos modelos estatísticos. 
QUADRO 1 - VARIÁVEIS DO MODELO E DESCRIÇÃO DA VARIÁVEL PARA OS TESTES AOS CARGOS DE DEPUTADO FEDERAL E ESTADUAL

\begin{tabular}{ll}
\hline & TESTE T1 \\
\hline Var. Dependente & IP_FIN_PTDO_CAND_NA_COLIGACAO (é o percentual de financiamento \\
& partidário recebido pelo candidato dentro da sua coligação) \\
\hline Var. Independente & DUMMY_ST_REELEICAO (Para esta variável Dummy foram dados os valores 1 \\
& para candidatos incumbentes e Zero para candidatos desafiantes); \\
& DUMMY_COR_RACA (Para esta variável Dummy foram dados os valores de 1 \\
& para candidatos que auto declararam a cor da pele como BRANCA e Zero para não \\
& brancos); \\
& DUMMY_GRAU_INSTRUCAO (Para esta variável Dummy foram dados os \\
& valores de 1 para os candidatos que declararam possuir ensino superior completo e \\
& Zero para os de menor grau de instrução); \\
& DUMMY_GENERO (Para esta variável Dummy foram dados os valores de 1 para \\
& candidatos do sexo masculino e Zero para as candidatas). \\
\hline Var. Dependente & TESTE T2 \\
\hline Var. Independente & IP_VOTO_CAND_NA_COL (é o percentual de votos conquistados pelo candidato \\
& em relação a soma total de votos de todos os candidatos dentro da mesma coligação); \\
\hline & IP_FIN_PTDO_CAND_NA_COLIGACAO (é o percentual de financiamento \\
& partidário recebido pelo candidato dentro da sua coligação) \\
\hline
\end{tabular}
FONTE: o autor (2020)

Nas Tabelas 2, 3, 4 e 5 são apresentados os resultados dos testes estatísticos T1 e T2 dentro das coligações de ambos os cargos. Os modelos não consideram para os cargos o universo de candidatos das disputas e sim apenas aqueles candidatos presentes dentro de cada coligação. Portanto, o teste T1 permite mensurar o grau de importância que os determinantes do financiamento têm em promover aos candidatos, o acesso ao Financiamento Partidário. Enquanto o T2, mensura a importância que o Financiamento Partidário tem em contribuir para a conquista de votos dentro da própria coligação. Tais análises só são possíveis quando ocorrem as seguintes condições, $\mathrm{N}$ de candidatos e valor de significância ${ }^{11}$.

Nesses testes não foi possível agregar o Coeficiente de Gini do Financiamento Partidário por Coligação, pois essa variável passa a se configurar em uma constante. Portanto, ao não variar, não afeta a variável dependente. Porém, nas tabelas será possível ler o Coeficiente para cada coligação, o $\mathrm{N}$ de cadeiras conquistadas pela coligação e a partir dessa leitura, tecer algumas conclusões.

Não obstante, lemos os dados que se seguem dando atenção ao valor do $R^{2}$, no qual assumiremos como grau de importância as seguintes condições: a) abaixo de ,100 muito fraco; b) ,100 ,300 fraco; c) ,300 ,700 médio; d) ,700 ,900 forte; e) acima de ,900 muito forte.

A Tabela 3 apresenta os testes sobre os determinantes do financiamento para o cargo de deputado federal. O teste aponta que variável com maior poder explicativo é aquela que se refere ao fato de o candidato concorrer à reeleição, ou seja, candidato incumbente. Em outras palavras, ser

\footnotetext{
${ }^{11}$ Para os testes realizados o F de Significância ( $p$-valor) é de $\mathrm{p}<0,05$.
} 
candidato à reeleição é o determinante que mais importa para o acesso ao Financiamento Partidário. Esse elemento está presente e é predominante em quatro das seis coligações analisadas. Na COL_8, não se faz presente pois, dos 41 candidatos, nenhum foi candidato à reeleição. Nesse caso, a variável gênero do candidato, sobretudo, ser homem, é a aquela que assume maior grau de importância. No caso da COL_10, a combinação entre as variáveis, candidato à reeleição e ser do sexo masculino, é o modelo com maior força explicativa para o acesso ao financiamento. Do contrário, ser candidato desafiante e do sexo feminino, é a combinação que promove menor acesso ao financiamento.

TABELA 2 - RESUMO DO TESTE T1 SOBRE OS DETERMINANTES DO ACESSO AO FINANCIAMENTO PARTIDÁRIO - (DEPUTADO FEDERAL)

\begin{tabular}{lrrrrrrrr}
\hline COL & MODELO & $\mathrm{R}$ & $\mathrm{R}^{2}$ & GINI & N_CAND & FIN_TOTAL & FIN_MÉDIO & CADEIRAS \\
\hline COL_1 & 1 &, $826 \mathrm{a}$ &, 682 &, 836 & 43 & $\mathrm{R} \$ 6.043 .635,93$ & $\mathrm{R} \$ 140.549,67$ & 3 \\
COL_4 & 1 &, $720 \mathrm{a}$ &, 519 &, 664 & 44 & $\mathrm{R} \$ 21.028 .861,27$ & $\mathrm{R} \$ 477.928,67$ & 8 \\
COL_7 & 1 &, $801 \mathrm{a}$ &, 641 &, 724 & 45 & $\mathrm{R} \$ 17.843 .073,72$ & $\mathrm{R} \$ 396.512,75$ & 10 \\
COL_8 & 1 &, $328 \mathrm{~d}$ &, 107 &, 901 & 41 & $\mathrm{R} \$ 290.200,00$ & $\mathrm{R} \$ 7.078,05$ & 3 \\
COL_10 & 1 &, $649 \mathrm{a}$ &, 422 &, 804 & 33 & $\mathrm{R} 4.120 .287,52$ & $\mathrm{R} \$ 124.857,20$ & 3 \\
COL_11 & 2 &, $704 \mathrm{c}$ &, 496 & & & & \\
\hline
\end{tabular}

a. Preditores: (Constante), DUMMY_ST_REELEICAO

b. Variável Dependente: IP_FIN_PTDO_CAND_NA_COLIGACAO

c. Preditores: (Constante), DUMMY_ST_REELEICAO, DUMMY_GENERO

d. Preditores: (Constante), DUMMY_GENERO

FONTE: O autor com dados do TSE por meio do software SPSS (2020)

A Tabela 3 apresenta os testes sobre o Financiamento Partidário e os votos do candidato para o cargo de deputado federal. Os dados apontam que apenas na COL_7, a relação entre financiamento e voto pode ser considerada fraca, mesmo sendo essa coligação a segunda em poderio financeiro e aquela que mais conquistou cadeiras. Nas demais coligações, o Financiamento Partidário é um elemento importante e contribui fundamentalmente para a conquista de votos. Vale neste caso apontar a COL_4, na qual o principal determinante que promove acesso ao financiamento (Tabela 2) foi o candidato concorrer à reeleição $\left(\mathrm{R}^{2}=, 519\right)$ e que o Financiamento Partidário tem importância $\left(\mathrm{R}^{2}=\right.$ ,549) na conquista de votos. Tal relação desperta interesse ao observar a Figura 5 em que o financiamento médio dos eleitos é superior a $\mathrm{R} \$ 1,1$ milhão, valor esse pelo menos três vezes maior que a média dos não eleitos, cerca de $\mathrm{R} \$ 330$ mil. de não eleitos. 
TABELA 3 - RESUMO DO TESTE T2 SOBRE FINANCIAMENTO PARTIDÁRIO E VOTO POR COLIGAÇÃO (DEPUTADO FEDERAL)

\begin{tabular}{lrrrrrrr}
\hline COL & $\mathrm{R}$ & $\mathrm{R}^{2}$ & GINI & N_CAND & FIN_TOTAL & FIN_MÉDIO & CADEIRAS \\
\hline COL_1 &, $884 \mathrm{a}$ &, 781 &, 836 & 43 & $\mathrm{R} \$ 6.043 .635,93$ & $\mathrm{R} \$ 140.549,67$ & \\
COL_2 &, $922 \mathrm{a}$ &, 851 &, 328 & 16 & $\mathrm{R} \$ 178.486,56$ & $\mathrm{R} \$ 11.155,41$ & 0 \\
COL_4 &, $741 \mathrm{a}$ &, 549 &, 664 & 44 & $\mathrm{R} \$ 21.028 .861,27$ & $\mathrm{R} \$ 477.928,67$ & \\
COL_5 &, $879 \mathrm{a}$ &, 772 &, 774 & 39 & $\mathrm{R} \$ 2.451 .818,27$ & $\mathrm{R} \$ 62.867,14$ & \\
COL_7 &, $510 \mathrm{a}$ &, 260 &, 724 & 45 & $\mathrm{R} \$ 17.843 .073,72$ & $\mathrm{R} \$ 396.512,75$ & 10 \\
COL_10 &, $923 \mathrm{a}$ &, 852 &, 804 & 33 & $\mathrm{R} \$ 4.120 .287,52$ & $\mathrm{R} \$ 124.857,20$ & 3 \\
COL_11 &, $950 \mathrm{a}$ &, 902 &, 829 & 40 & $\mathrm{R} \$ 1.932 .069,16$ & $\mathrm{R} \$ 48.301,73$ & \\
\hline
\end{tabular}

a. Preditores: (Constante), IP_FIN_PTDO_CAND_NA_COLIGACAO

b. Variável Dependente: IP_VOTO_CAND_NA_COL

* Sig à $\mathrm{p}<0,05$

FONTE: O autor com dados do TSE por meio do software SPSS (2020)

A Tabela 4 apresenta os testes sobre os determinantes do financiamento para o cargo de deputado estadual. Os dados apontam que a relação é diferente das disputas para o cargo de deputado federal. Ser candidato à reeleição não é condição tão importante para o acesso ao financiamento, ainda que essa se apresente nas coligações COL_1, COL_5, COL_7 e COL_12, porém com um R² médio apenas na COL_1. No caso da COL_9, a combinação que melhor explica o acesso ao financiamento é primeiramente o candidato ser do sexo masculino e, segundamente, combinado com o gênero do candidato, concorrer à reeleição, mas ainda com um grau baixo de determinação. No entanto, note que essa é a coligação com maior aporte do Financiamento Partidário e a que mais conquistou cadeiras, totalizando 10. Uma outra condição que chama a atenção é que, ao visualizar a Figura 6 (no apêndice), a média do Financiamento Partidário dos candidatos eleitos é menor que a média dos não eleitos. Afinal, a que se deve esse fenômeno?

TABELA 4 - RESUMO DO TESTE T1 SOBRE OS DETERMINANTES DO ACESSO AO FINANCIAMENTO PARTIDÁRIO - (DEPUTADO ESTADUAL)

\begin{tabular}{lrrrrrrrr}
\hline COL & MODELO & $\mathrm{R}$ & $\mathrm{R}^{2}$ & GINI & N_CAND & FIN_TOTAL & FIN_MÉDIO & CADEIRAS \\
\hline COL_1 & 1 &, $671 \mathrm{a}$ &, 450 &, 664 & 25 & $\mathrm{R} \$ 1.614 .263,15$ & $\mathrm{R} \$ 70.185,35$ & 2 \\
COL_3 & 1 &, $916 \mathrm{c}$ &, 838 &, 763 & 36 & $\mathrm{R} \$ 10.333,31$ & $\mathrm{R} \$ 295,24$ & 1 \\
COL_5 & 1 &, $334 \mathrm{a}$ &, 111 &, 642 & 45 & $\mathrm{R} \$ 1.201 .277,06$ & $\mathrm{R} \$ 28.601,83$ & 3 \\
COL_6 & 1 &, $389 \mathrm{~d}$ &, 152 &, 618 & 62 & $\mathrm{R} \$ 2.794 .038,91$ & $\mathrm{R} \$ 47.356,59$ & 3 \\
COL_7 & 1 &, $331 \mathrm{a}$ &, 109 &, 875 & 73 & $\mathrm{R} \$ 1.958 .629,98$ & $\mathrm{R} \$ 27.980,43$ & 3 \\
COL_9 & 1 &, $473 \mathrm{c}$ &, 223 &, 666 & 68 & $\mathrm{R} \$ 4.775 .767,66$ & $\mathrm{R} \$ 82.340,82$ & 10 \\
COL_12 & 2 &, $530 \mathrm{e}$ &, 281 & & & & \\
COL_14 & 1 &, $477 \mathrm{a}$ &, 227 &, 719 & 31 & $\mathrm{R} \$ 790.821,68$ & $\mathrm{R} \$ 29.289,69$ & \\
\hline
\end{tabular}

a. Preditores: (Constante), DUMMY_ST_REELEICAO

b. Variável Dependente: IP_FIN_PTDO_CAND_NA_COLIGACAO

c. Preditores: (Constante), DUMMY_GENERO

d. Preditores: (Constante), DUMMY_GRAU_INSTRUCAO

e. Preditores: (Constante), DUMMY_GENERO, DUMMY_ST_REELEICAO

FONTE: O autor com dados do TSE por meio do software SPSS (2020) 
A Tabela 5 Tabela 4apresenta os testes sobre o Financiamento Partidário e os votos do candidato para o cargo de deputado estadual. Observamos ao menos dois fenômenos bastante curiosos e que se referem à COL_4 e COL_10. A primeira, apresenta $\mathrm{R}^{2}$ muito fraco. A segunda, $\mathrm{R}^{2}$ muito forte. São as duas coligações que mais conquistaram cadeiras (dentro dos casos testados), 14 e 8 respectivamente. O aporte financeiro é, para a COL_4, cerca de 13 vezes maior que a da COL_10. Ambas apresentam uma maneira de distribuição do financiamento bem diferentes, sendo Gini = ,793 e ,956, respectivamente. Porém, irrelevante, se considerarmos a discrepância no montante de Financiamento Partidário envolvido. Ademais, candidatos eleitos são mais bem financiados que não eleitos. No entanto, a média de Financiamento Partidário dos candidatos não eleitos da COL_4 é superior que a dos eleitos da COL_10.

Dito isso, ambas as coligações não apresentaram resultados significativos quanto aos determinantes do financiamento (Tabela 4). Ou seja, a prática da distribuição de recursos financeiros não segue a lógica de que, concorrer à reeleição, é um determinante no acesso ao financiamento. Isso pode indicar que as estratégias para o sucesso eleitoral dessas duas coligações diferem bastante das demais candidaturas para deputados estaduais, e que no caso da COL_10, o sucesso eleitoral não passa pelo Financiamento Partidário, enquanto no caso da COL_4, apesar do montante bastante superior, tal financiamento pode ser menos determinante para as estratégias.

TABELA 5 - RESUMO DO TESTE T2 SOBRE FINANCIAMENTO PARTIDÁRIO E VOTO POR COLIGAÇÃO (DEPUTADO ESTADUAL)

\begin{tabular}{lrrrrrrr}
\hline COL & $\mathrm{R}$ & $\mathrm{R}^{2}$ & GINI & N_CAND & FIN_TOTAL & FIN_MÉDIO & CADEIRAS \\
\hline COL_1 &, $736 \mathrm{a}$ &, 542 &, 664 & 25 & $\mathrm{R} \$ 1.614 .263,15$ & $\mathrm{R} \$ 70.185,35$ & 2 \\
COL_4 &, $232 \mathrm{a}$ &, 054 &, 793 & 79 & $\mathrm{R} \$ 7.085 .689,68$ & $\mathrm{R} \$ 109.010,61$ & 14 \\
COL_5 &, $699 \mathrm{a}$ &, 488 &, 642 & 45 & $\mathrm{R} \$ 1.201 .277,06$ & $\mathrm{R} \$ 28.601,83$ & 3 \\
COL_6 &, $538 \mathrm{a}$ &, 289 &, 618 & 62 & $\mathrm{R} \$ 2.794 .038,91$ & $\mathrm{R} \$ 47.356,59$ & 3 \\
COL_7 &, $514 \mathrm{a}$ &, 265 &, 875 & 73 & $\mathrm{R} \$ 1.958 .629,98$ & $\mathrm{R} \$ 27.980,43$ & 3 \\
COL_10 &, $985 \mathrm{a}$ &, 971 &, 956 & 65 & $\mathrm{R} \$ 548.828,32$ & $\mathrm{R} \$ 9.628,57$ & 8 \\
COL_12 &, $862 \mathrm{a}$ &, 743 &, 719 & 31 & $\mathrm{R} \$ 790.821,68$ & $\mathrm{R} \$ 29.289,69$ & \\
\hline
\end{tabular}

a. Preditores: (Constante), IP_FIN_PTDO_CAND_NA_COLIGACAO

b. Variável Dependente: IP_VOTO_CAND_NA_COL

FONTE: O autor com dados do TSE por meio do software SPSS (2020)

\section{RESULTADOS}

Os resultados da exploração dos dados no recorte estudado apontam que, maior ou menor aporte financeiro, não reflete de maneira direta o modo como o recurso é distribuído. Ou seja, ter muito Financiamento Partidário não implica necessariamente em dividi-lo de maneira mais equânime. 
Da mesma forma, ter pouco, não significa destiná-lo a um número reduzido de candidatos. No entanto, sugere que o aporte financeiro e o número de candidaturas competitivas (considerando candidatos incumbentes) são elementos fundamentais para a apreciação de estratégias que visem potencializar as chances de conquistas de cadeiras.

Ao analisar os dados apresentados, o Coeficiente de Gini sugere que, a distribuição mais equânime do Financiamento Partidário pelas coligações que detêm maior quantia desse recurso nas disputas ao cargo de deputado federal, é a estratégia que melhor possibilita o sucesso eleitoral da coligação. Por outro lado, nas disputas ao cargo de deputado estadual, maior quantia de Financiamento Partidário associado a distribuição, conforme indica o Coeficiente de Gini, não se revela em uma estratégia mais assertiva, visto o caso das coligações COL_4 e COL_10 que possuem montantes bastante diferentes de Financiamento Partidário e relevante sucesso eleitoral.

Os dados da análise estatística revelam que, elementos determinantes do financiamento, especialmente a condição do candidato concorrer à reeleição, é a variável que proporciona melhor acesso ao Financiamento Partidário, ao menos para o cargo de deputado federal. Da mesma forma, o Financiamento Partidário assume um papel fundamental ao contribuir para a conquista de votos dentro da própria coligação.

Para o cargo de deputado estadual, os dados apontam que, não necessariamente, concorrer à reeleição é o principal determinante do acesso ao Financiamento Partidário. Do mesmo modo, o financiamento não é fundamental para o sucesso dentro da coligação, pelo menos não de forma tão dominante como ocorre na disputa para deputado federal. Mediante a essa condição, acredita-se que as modalidades de financiamento privado, bem como elementos exógenos ao controle das coligações, podem contribuir em maior grau para a conquista do voto. Isso sugere uma investigação diferenciada sobre quais elementos do perfil do candidato e modalidades de financiamento importam mais para as disputas ao cargo de deputado estadual.

\section{CONSIDERAÇÕES FINAIS}

A exploração dos dados nos permite levantar algumas questões sobre o objeto estudado. No entanto, revela que há limites que precisam ser superados no que se refere à metodologia e técnicas de análise que foram utilizadas. Afinal, se uma das contribuições deste material é o de trazer a aplicação do Coeficiente de Gini para a análise da concentração/distribuição do Financiamento 
Partidário, de que maneira ele pode ser melhor aproveitado? Além do mais, que elementos poderiam ser trazidos para o aprimoramento das análises e dos resultados possibilitando melhor conhecimento do fenômeno?

Entendemos que a discrepância entre os montantes envolvidos é bastante expressiva entre as coligações e ao que tudo indica, ambos os cargos ignoram questões relativas ao espectro ideológico dos partidos. Acreditamos que tal elemento deve ser agregado e pontuado nas análises futuras.

No que se refere às estratégias dos partidos, visando maior sucesso eleitoral, cabe questionar se: a soma de recursos financeiros pelos partidos é elemento central no ato da construção das coligações? Pressupondo que os partidos somam esforços de maneira estratégica para o alcance de cadeiras, e sabem do papel fundamental do dinheiro em uma campanha, organizar-se a partir do quadro absoluto de recursos financeiros é o que motiva as alianças? Assim sendo, é preciso pensar no cálculo de sucesso que centraliza elementos dos candidatos como incumbência e detenção de capital político. Não obstante, quais aspectos da conjuntura política poderiam explicar as coligações? Estar nas coligações de apoio ao governo do Estado importam para o acesso ao financiamento e sucesso eleitoral?

O volume aportado importa para a escolha de distribuir mais ou menos? A melhor distribuição do recurso é a estratégia para o sucesso? A distribuição permite que mais candidatos façam votos e assim alimentem a legenda, o que permite maior alcance do Quociente Eleitoral (QE) para acesso as cadeiras? Ou a melhor estratégia seria a da concentração dos recursos e apostas em perfis determinados?

No que se refere especificamente ao financiamento, é preciso explorar de forma mais pormenorizada como os recursos financeiros transitam entre os partidos das coligações ou seriam as doações dos partidos das coligações exclusivamente para os candidatos do partido?

Por fim, que elementos contribuem para pensar no cenário político futuro, no qual a partir das eleições de 2020, não será mais permitido aos partidos políticos se coligarem para disputarem cadeiras nas proporcionais.

\section{REFERÊNCIAS}

BOLOGNESI, Bruno et al. Como os partidos disbribuem o dinheiro: estrutura organizacional e recursos eleitorais em 2014 no BrasilXI Encontro ABCP. [S. l.: s. $n$. .]. Disponível em: https://doi.org/10.1017/CBO9781107415324.004.

BORBA, Felipe; CERVI, Emerson Urizzi. Relação entre propaganda, dinheiro e avaliação de 
governo no desempenho de candidatos em eleições majoritárias no Brasil. Opinião Pública, [S. l.], v. 23, n. 3, p. 754-785, 2017. Disponível em: https://doi.org/10.1590/1807-01912017233754. Acesso em: 21 jun. 2019.

CERVI, Emerson Urizzi. Financiamento de campanhas e desempenho eleitoral no Brasil: análise das contribuições de pessoas físicas, jurídicas e partidos políticos às eleições de 2008 nas capitais de Estado. Revista Brasileira de Ciência Política, Brasília, n. 4, p. 135-167, 2010.

GEARA, Luiz Eduardo et al. O financiamento eleitoral nas comissões permanentes da Câmara dos Deputados: uma análise do meta relacionamento entre empresas e parlamentares. E-legis, Brasília, p. 60-81, 2018.

HOROCHOVSKI, Rodrigo Rossi et al. Redes de partidos políticos tecidas por financiadores: um estudo das eleições de 2010 no Brasil. Teoria \& Sociedade, [S. l.], v. 23, n. 2, p. 49-78, 2015. Disponível em: http://www.din.uem.br/sbpo/sbpo2016/pdf/156426.pdf

HOROCHOVSKI, Rodrigo Rossi et al. Estruturas de poder nas redes de financiamento político nas eleições de 2010 no Brasil. Opinião Pública, Campinas, v. 22, n. 1, p. 28-55, 2016. Disponível em: https://doi.org/10.1590/1807-0191201622128. Acesso em: 21 jun. 2019.

HOROCHOVSKI, Rodrigo Rossi; JUNCKES, Ivan Jairo; ZELINSKI, Luiz Fernando. Financiamento dos candidatos nas eleições de 2016 em Curitiba. In: BOLOGNESI, Bruno; ROEDER, Karolina; BABIRESKI, Flávia (org.). Quem decide concorrer: a eleição e os vereadores em Curitiba. Curitiba: TRE-PR: Massimo Editoral, 2018. p. 151-174. E-book.

JUNCKES, Ivan Jairo et al. POWER AND DEMOCRACY: AN ANALYSIS ON THE ELECTORAL FINANCING NETWORK IN 2014 IN BRAZIL PODER E DEMOCRACIA: UMA ANÁLISE DA REDE DE FINANCIAMENTO ELEITORAL EM 2014 NO BRASIL TT - Pouvoir et dÉmocratie: une analyse du rÉseau de financement Électoral en 2014. Revista Brasileira de Ciências Sociais, [S. l.], v. 34, n. 100, p. e3410006-e3410006, 2019. Disponível em: https://doi.org/10.1590/3410006/2019. Acesso em: 18 fev. 2019.

MANCUSO, Wagner Pralon. Investimento eleitoral no Brasil: balanço da literatura (2001-2012) e agenda de pesquisa. Revista de Sociologia e Política, [S. l.], v. 23, n. 54, p. 155-183, 2015. Disponível em: https://doi.org/10.1590/1678-987315235409. Acesso em: 19 jul. 2019.

MANCUSO, Wagner Pralon; HOROCHOVSKI, Rodrigo Rossi; CAMARGO, Neilor Fermino. Financiamento eleitoral empresarial direto e indireto nas eleições nacionais de 2014. Revista Brasileira de Ciência Política, $[S$. l.], n. 27, p. 9-36, 2018. Disponível em: https://doi.org/10.1590/0103-335220182701. Acesso em: 26 mar. 2019.

MANCUSO, Wagner Pralon; SPECK, Bruno Wilhelm. Financiamento empresarial na eleição para Deputado Federal (2002-2010): Determinantes e consequências. Revista Teoria \& Sociedade, [S. l.], v. 23, n. 2, p. 103-125, 2015 a.

MANCUSO, Wagner Pralon; SPECK, Bruno Wilhelm. Os preferidos das empresas: um estudo de determinantes do financiamento eleitoral empresarial. In: COSTA, Paulo Roberto Neves; PONT, Juarez Varallo (org.). Empresários, Desenvolvimento, Cultura e Democracia. [S. l.]: Editora 
UFPR, 2015 b. p. 217-229. E-book. Disponível em: http://www.tse.jus.br/eleicoes/repositorio-dedados-eleitorais. Acesso em: 10 mar. 2020.

SACCHET, Teresa; SPECK, Bruno Wilhelm. Financiamento eleitoral, representação política e gênero: uma análise das eleições de 2006. Opinião Pública, [S. l.], v. 18, n. 1, p. 177-197, 2012. Disponível em: https://doi.org/10.1590/S0104-62762012000100009. Acesso em: 23 jan. 2019.

SAMUELS, David. Does Money Matter? Credible Commitments and Campaign Finance in New Democracies: Theory and Evidence from Brazil. Comparative Politics, [S. l.], v. 34, n. 1, p. 23, 2001. Disponível em: https://doi.org/10.2307/422413

SILVA, Edson Armando et al. Mulheres nas Eleições de 2014 no Brasil: Evidências de Exclusão e Marginalização Política. Revista Latino-americana de Geografia e Genero, [S. l.], v. 8, n. 1, p. 251-272, 2017. Disponível em: https://doi.org/10.5212/rlagg.v.8.i1.0015

SPECK, Bruno Wilhelm; CERVI, Emerson Urizzi. Dinheiro, Tempo e Memória Eleitoral: Os Mecanismos que Levam ao Voto nas Eleições para Prefeito em 2012. Dados, Rio de Janeiro, v. 59, n. 1, p. 53-90, 2016. Disponível em: https://doi.org/10.1590/00115258201671. Acesso em: 21 jun. 2019.

SPECK, Bruno Wilhelm; MANCUSO, Wagner Pralon. A study on the impact of campaign finance, political capital and gender on electoral performance. Brazilian Political Science Review, [S. l.], v. 8, n. 1, p. 34-57, 2014. Disponível em: https://doi.org/10.1590/1981-38212014000100002. Acesso em: 15 jul. 2019.

ZELINSKI, Luiz Fernando; EDUARDO, Maria Cecília. As novas regras do financiamento eleitoral de campanhas nas eleições de 2018 e seus efeitos nas candidaturas de homens e mulheres para o cargo de Deputado Estadual no Estado do Paraná. Revista do Legislativo Paranaense, [S. l.], n. 3, p. 2952 2019.

Disponível

em: http://revista.alep.pr.gov.br/index.php/escolalegislativo/article/view/89/pdf. Acesso em: 2 set. 2019.

\section{APÊNDICE}


I Seminário Discente de Ciência Política (SDCP)

Programa de Pós-Graduação em Ciência Política (PPGCP)

Universidade Federal do Paraná (UFPR)

Curitiba, 20 a 21 de agosto de 2020

FIGURA 1 - GINI DO FINANCIAMENTO PARTIDÁRIO POR COLIGAÇÃO EM VALORES ABSOLUTOS E MÉDIO (CARGO DE DEPUTADO FEDERAL)

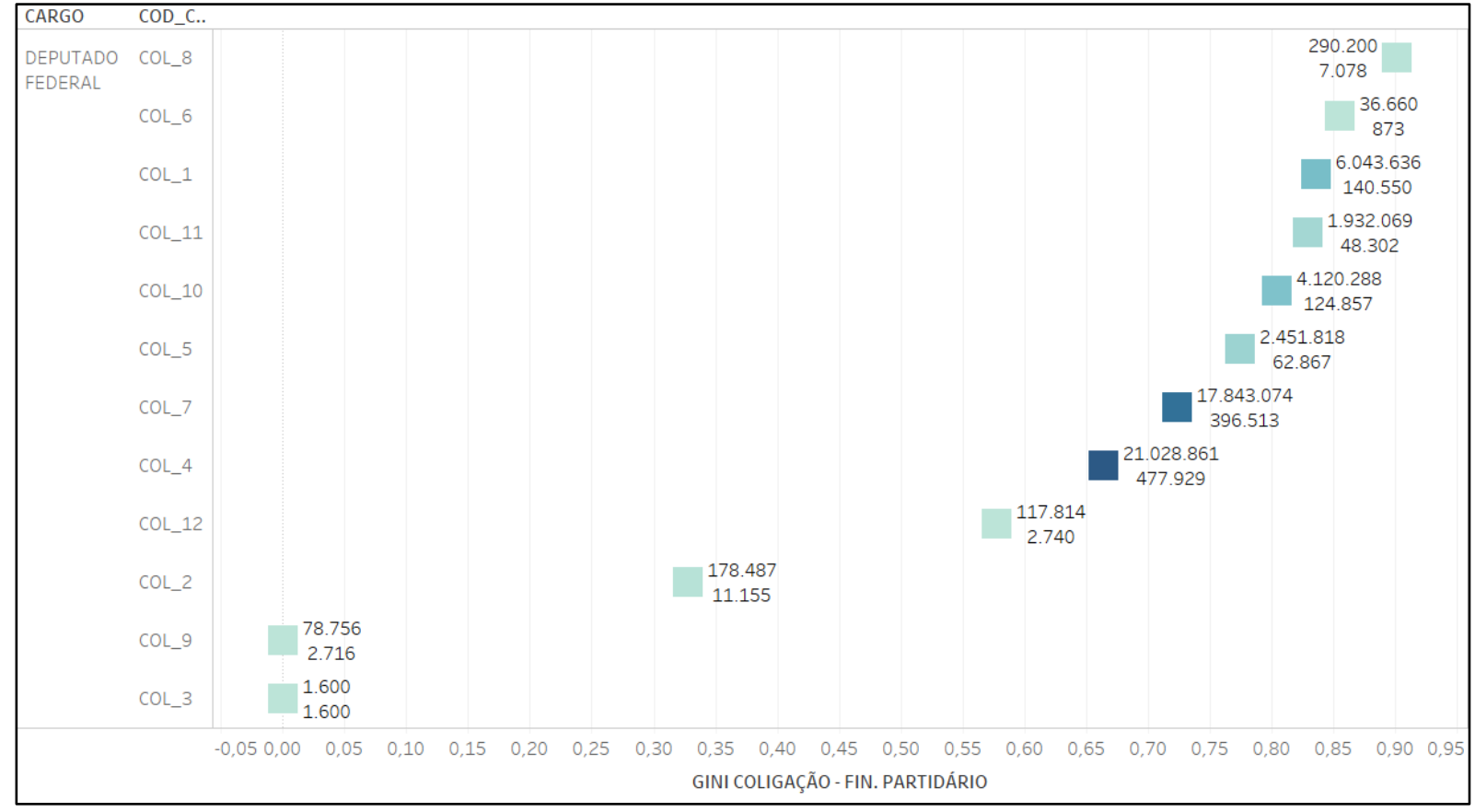

FONTE: O autor com dados do TSE por meio do software Tableau (2020)

FIGURA 2 - GINI DO FINANCIAMENTO PARTIDÁRIO POR COLIGAÇÃO EM VALORES ABSOLUTOS E MÉDIO (CARGO DE DEPUTADO ESTADUAL)

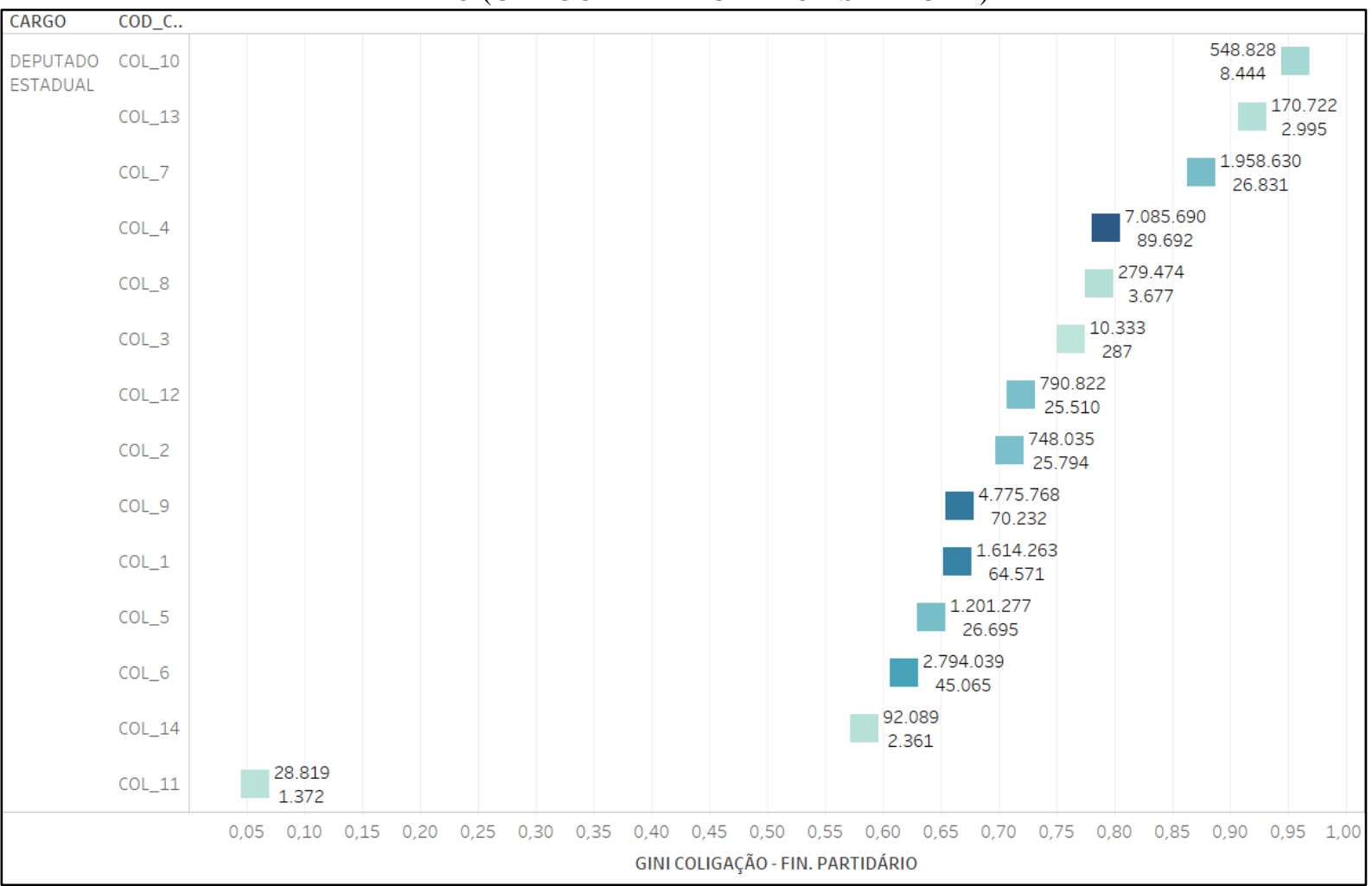

FONTE: O autor com dados do TSE por meio do software Tableau (2020) 
FIGURA 3 - NÚMERO DE CADEIRAS CONQUISTADAS POR COLIGAÇÃO X COEFICIENTE DE GINI DO FINANCIAMENTO PARTIDÁRIO PARA O CARGO DE DEPUTADO FEDERAL

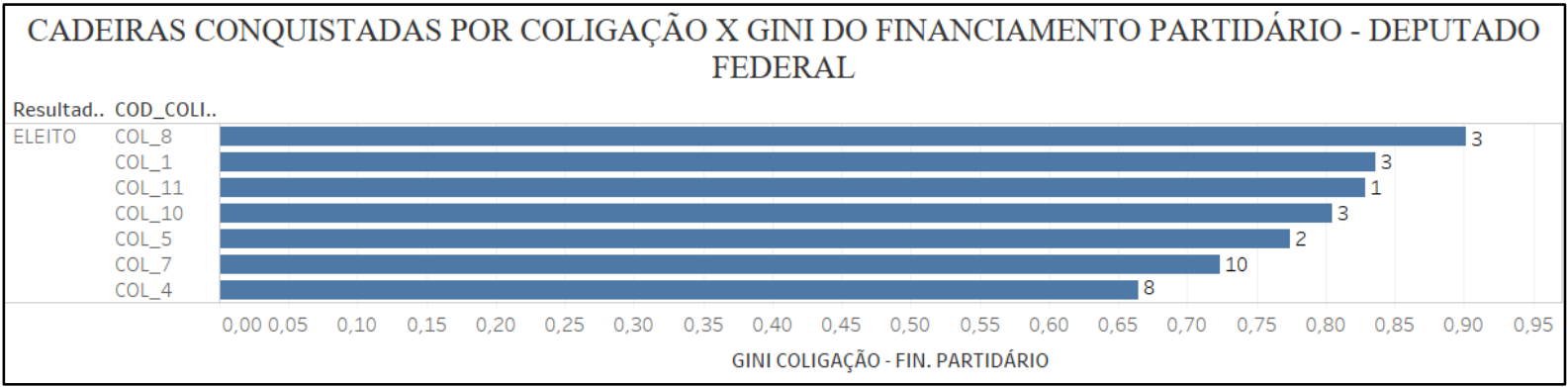

FONTE: O autor com dados do TSE por meio do software Tableau (2020)

FIGURA 4 - NÚMERO DE CADEIRAS CONQUISTADAS POR COLIGAÇÃO X COEFICIENTE DE GINI DO FINANCIAMENTO PARTIDÁRIO PARA O CARGO DE DEPUTADO ESTADUAL

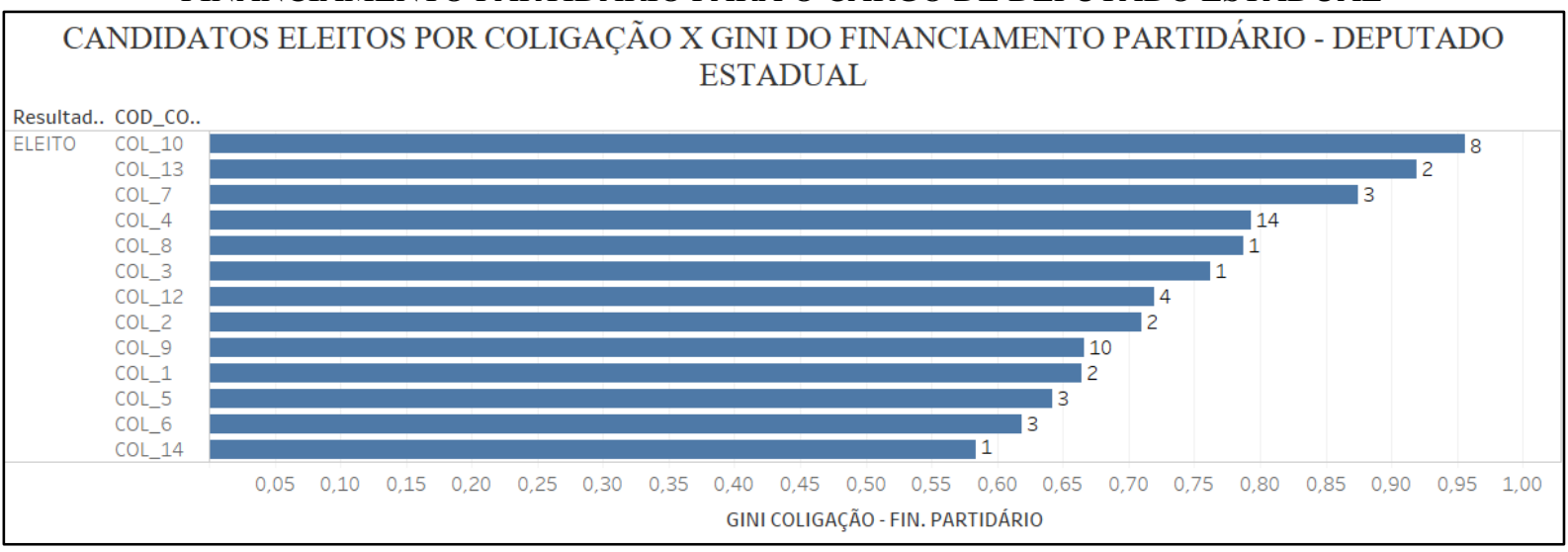

FONTE: O autor com dados do TSE por meio do software Tableau (2020)

FIGURA 5 - FINANCIAMENTO PARTIDÁRIO MÉDIO ENTRE ELEITOS E NÃO ELEITOS POR COLIGAÇÃO

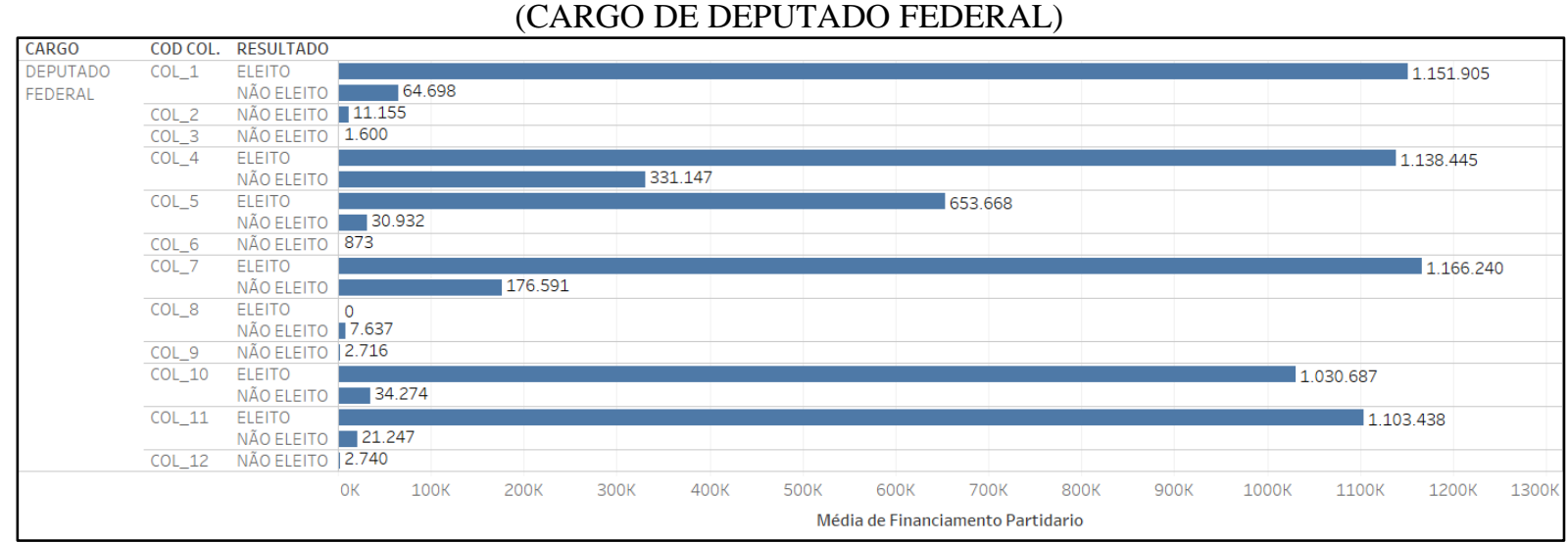

FONTE: O autor com dados do TSE por meio do software Tableau (2020) 
I Seminário Discente de Ciência Política (SDCP) Programa de Pós-Graduação em Ciência Política (PPGCP)

Universidade Federal do Paraná (UFPR) Curitiba, 20 a 21 de agosto de 2020

FIGURA 6 - FINANCIAMENTO PARTIDÁRIO MÉDIO ENTRE ELEITOS E NÃO ELEITOS POR COLIGAÇÃO (CARGO DE DEPUTADO ESTADUAL)

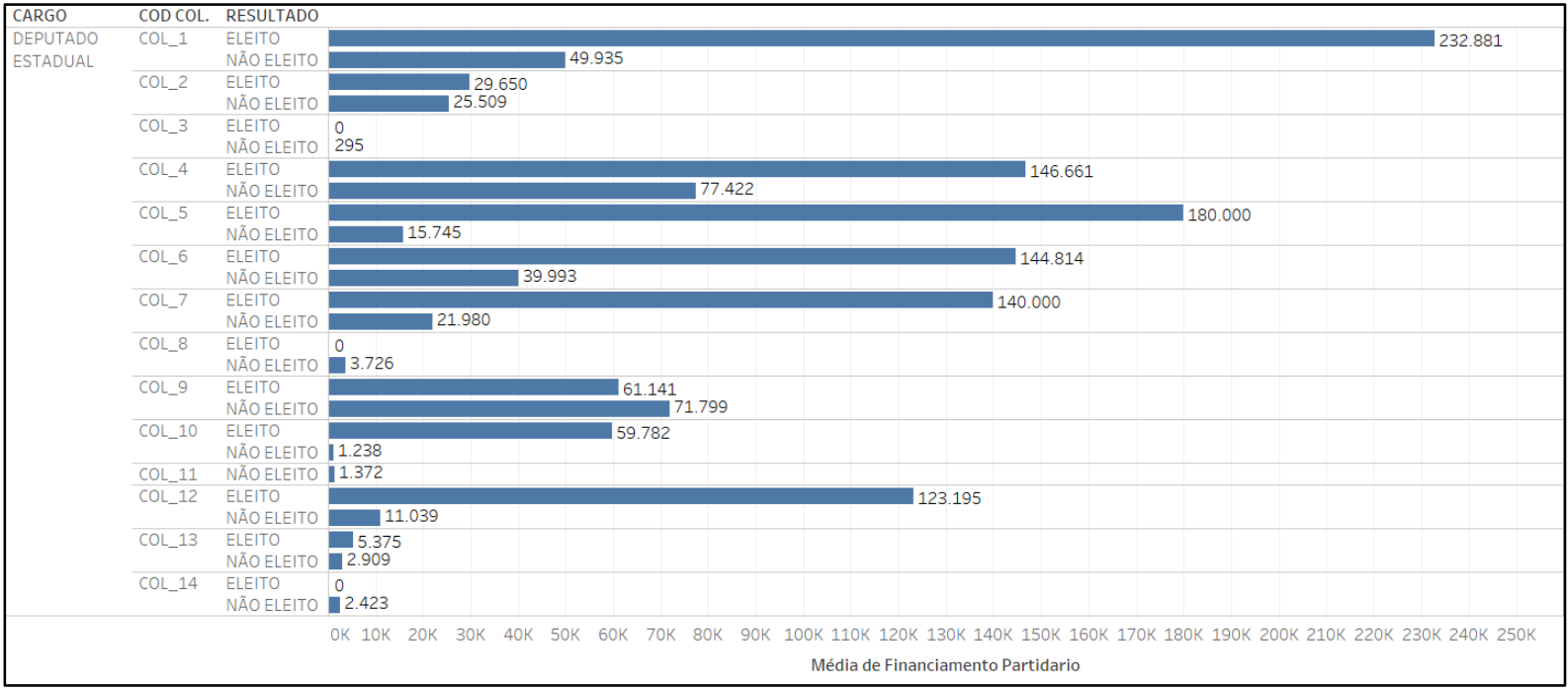

FONTE: O autor com dados do TSE por meio do software Tableau (2020) 